\title{
Cost Effective Speed Measurement using Optical Method for Belt Conveyors
}

\author{
Emmanuel Gospel Raj Rivington*, T. A. Selvan, Bhuvaneswari Mani, Sabari Kannan Muthalagu \\ and Mouliha Sree Subbian Veluswaami \\ Department of Mechatronics Engineering, Sri Krishna College of Engineering and Technology, \\ Coimbatore-641008,Tamil Nadu, India;emmanuelgospelraj@skcet.ac.in, selvanta@skcet.ac.in, \\ bhuvaneswarim@skcet.ac.in,15eumt090@skcet.ac.in,15eumt071@skcet.ac.in
}

\begin{abstract}
Objectives: The aim is to replace the current shaft encoders with a non-contact and cost effective optoelectronic type of speed measurement in the conveyor setup. Methods/Statistical Analysis: There are several methods by which the RPM of the motor can be measured. Existing conveyors uses Shaft encoder for measuring the speed of the conveyor. The main drawback of these encoders is, they are used in contact with the conveyors. This reduces the lifespan of the conveyor belt because of wear of the belt caused by the contact of the encoder wheel. Findings: Initially, different types of encoders were studied and magnetic encoders placed on the motor shaft were found as the right choice, but it was found to be costly. On the other hand, IR emitter and receiver when placed opposite to the rotating disk that contains alternate black and white patterns were found effective in measuring the roller speed. The alternative black and white coloured pattern was chosen because of the property of IR Light to be absorbed by the black region and to be totally reflected by the white surface. It is also inferred from the experiment that the error percentage of the mouse sensor was negligible when the air gap between the IR LEDs and the pattern is less than $20 \mathrm{~mm}$. Application/Improvements: All earlier available encoder devices were in contact with the conveyor setup in one way or the other and are expensive. However, the proposed idea of using IR LEDs to replace the encoder for speed measuring is both contactless and cost effective solution. Placement of suitable lens in front of the IR emitter and receiver will increase the measuring distance without compromising on the reliability of the conveyor speed output.
\end{abstract}

Keywords: Conveyor, Infrared Emission, Optoelectronics, RPM, Shaft Encoder, Speed Measurement

\section{Introduction}

An innovative cost effective method of speed sensing for industrial belt conveyor application is proposed in this paper. The speed measurement system consists of IR $(I R=$ Infrared $)$ emitter and receiver that will utilize a circular pattern that consist of a pattern of alternate black and white strips on one of the one side of the roller to sense the speed of the conveyor. The IR sensor will be placed directly opposite to the pattern in such a way that the IR emitter and receiver face the pattern. The basic principle behind is that IR light will be totally reflected when by the white surface and it is totally absorbed by the black surface. The RPM sensor ${ }^{1}$ counts the number of white strips in order to measure the RPM or speed of the conveyor.

\section{Materials and Methods}

The following components were used for the implementation of this method as shown in Figures 1 and 2:

- Attiny85 (Microcontroller).

- IR emitter and receiver.

- LCD.

- I2C backpack for LCD.

- LM 358 operational amplifier. 


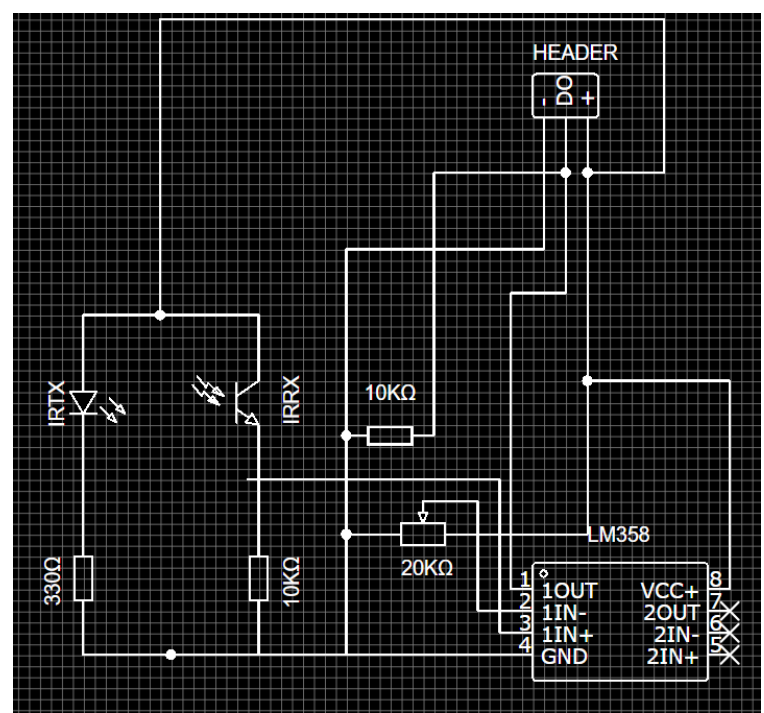

Figure 1. Sensor circuit diagram.

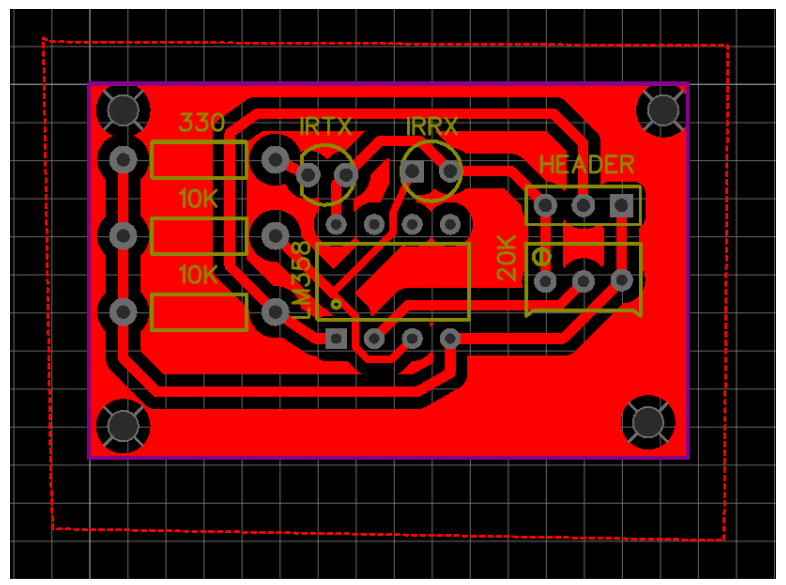

Figure 2. PCB layout.

\section{Methods}

\subsection{Characteristics of Shaft Encoders}

It is very essential to replace the shaft encoders that are currently used in speed measurement in conveyors with non-contact solution because in due course of time, the available shaft encoders cause wear and tear of the belt conveyor. Except roller shaft mount and surface mount encoders, the other type of encoders such as motor mount, belt chain mount are not reliable because of slippage of belt conveyor and other factors. In case of motor mount encoders ${ }^{2}$, the mechanical tolerances in the mechanical components of the conveyor reduce the level of feedback when compared with other type of encoders. In belt/chain driven mount encoder ${ }^{2}$, stack errors occur because of mechanical tolerance of components and it reduces the life span of the conveyor because of the radial load applied to the encoder by the belt or chain. Surface mount type of encoders ${ }^{2}$ produce the accurate speed measurement since it eliminates the mechanical tolerances. However, the main drawback here is that, in surface mount, the encoder wheel will be rotated on the surface of the conveyor belt. This in turn reduces the life of the conveyor belt causing wear and tear of the belt. The pattern is therefore placed on the roller of the conveyor.

\subsection{Selection of Pattern}

Each time the infrared emitter emits infrared light, the light gets totally reflected, when the emitted light hits the white surface and is totally absorbed when the emitted light hits the black surface. Therefore, there will be a high pulse when the infrared light is reflected from the white surface and a low pulse when the infrared light is reflected from the black surface as shown in Figure 3. The transition in the pulse from high to low during the rotation of the wheel from white to black surface and from low to high during the shift from black to white surface is detected and counted as shown in Figure 4.

The pattern developed consists of alternate black and white strips arranged radially in a circular disc. 30 White and 30 Black strips are placed alternately on the pattern. The number of pulses per rotation will be equal to 30 . Therefore the speed of the roller in RPM $=$ No. of pulses/30 as shown in Figures 5 and 6.

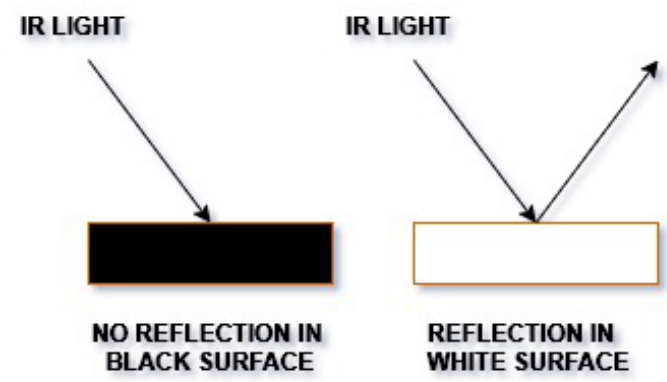

Figure 3. IR light property.

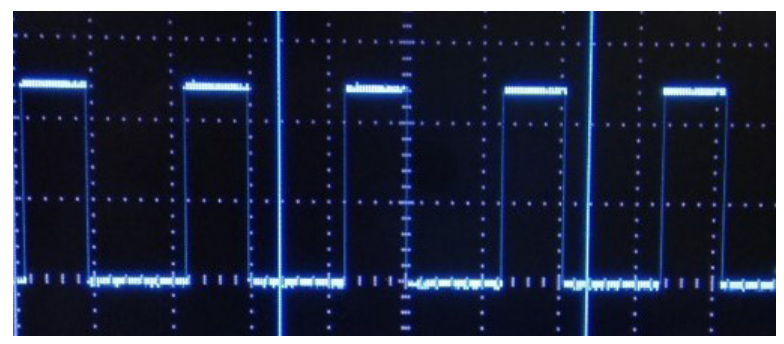

Figure 4. PWM output signal of the sensor. 


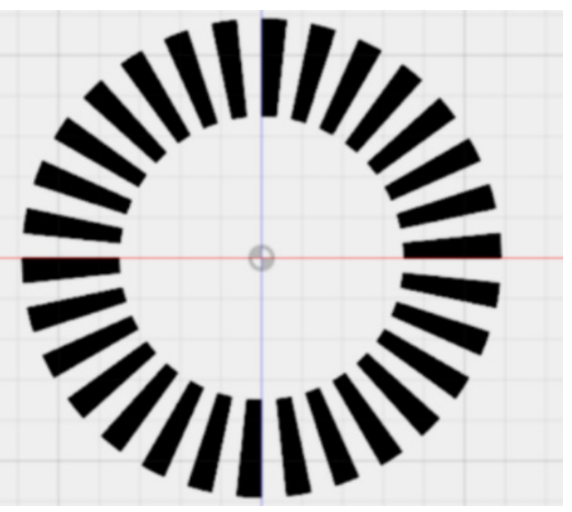

Figure 5. Pattern block diagram.

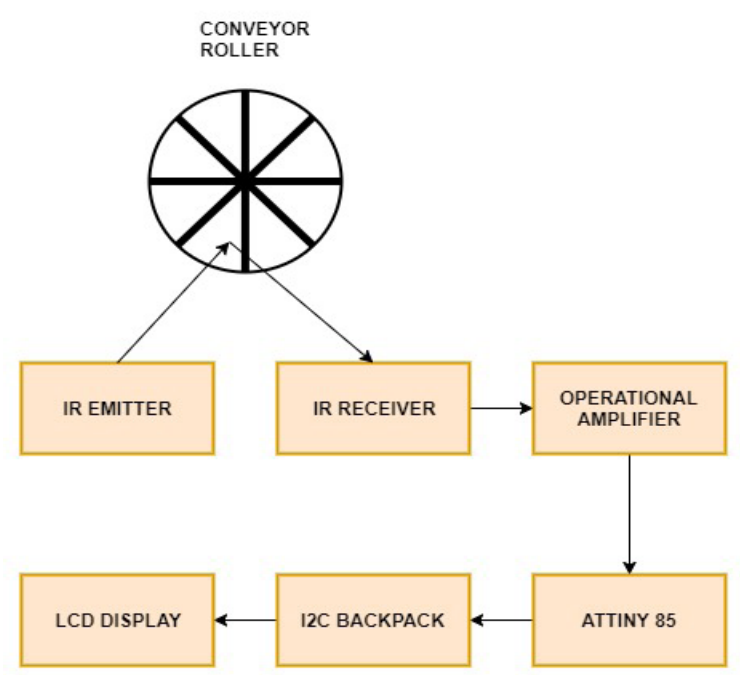

Figure 6. Block diagram.

\section{Control of VFD}

Drives $^{3}$ such as AC drive and DC drive are widely used in industries. Variable Frequency Drive (VFD) is used to control speed ${ }^{4}$ of AC motor by varying the power supply frequency. They have two modes of control namely remote mode and local mode. The local mode is used to control the speed of the motor by the operator, manually using potentiometer given on the device whereas in the remote mode, the connection wiring will be done in the external ports given in bottom of the drive. In both the cases, usually a DC voltage in the range $0 \mathrm{~V}$ to $10 \mathrm{~V}$ is used to control the frequency and hence the speed of the drive $e^{5}$. At $10 \mathrm{~V}$ control voltage, the motor runs at its maximum RPM and when the control voltage is reduced, speed reduces proportionately and reaches zero when the control voltage becomes $0 \mathrm{~V}$.
The proposed RPM sensor is found compatible with all industrial drives. Though it produced $0 \mathrm{~V}$ to $5 \mathrm{~V}$ at its output, using amplifier circuit with gain value two, a range from $0 \mathrm{~V}$ to $10 \mathrm{~V}$ was obtained. Thus it was easily interfaced with a VFD drive whereas in shaft encoders, it would be difficult to interface with an existing drive as shown in Figure 7.

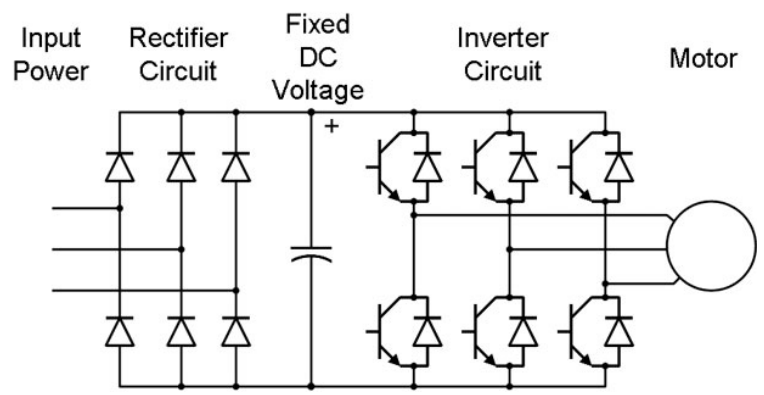

Figure 7. VFD circuit.

\section{Results}

The speed measured by this RPM sensor was reliable with minimal error percentage at the same time being cost effective. The results obtained through experimentation with IR emitter and receiver based sensor is shown in Table 1.

Table 1. Speed measurement readings

\begin{tabular}{|c|c|c|c|}
\hline S. No. & $\begin{array}{l}\text { Actual Speed } \\
\text { (RPM) }\end{array}$ & $\begin{array}{l}\text { Measured Speed } \\
(\text { RPM })\end{array}$ & $\begin{array}{l}\text { Error } \\
\text { Percentage }\end{array}$ \\
\hline 1 & 100 & 100 & 0.00 \\
\hline 2 & 200 & 199 & -0.50 \\
\hline 3 & 300 & 301 & 0.33 \\
\hline 4 & 400 & 399 & -0.25 \\
\hline 5 & 500 & 500 & 0.00 \\
\hline 6 & 600 & 599 & -0.17 \\
\hline 7 & 700 & 701 & 0.14 \\
\hline 8 & 800 & 800 & 0.00 \\
\hline 9 & 900 & 899 & -0.11 \\
\hline 10 & 1000 & 1003 & 0.30 \\
\hline
\end{tabular}

\section{Discussion}

Existing conveyors use shaft encoder for measuring the speed of the conveyor. The main drawback of these encoders is, they are used in contact with the conveyors. This reduces the lifespan of the conveyor belt because of 
wear of the belt caused by the contact of the encoder wheel. The proposed method is a cost effective replacement to shaft encoder for speed measurement in conveyors with zero or minimal error. The domain that we have used for speed measurement is Optoelectronics since it will pave way for non-contact way of speed measurement.

\section{Conclusion}

With Infrared emitter and receiver based sensor, a noncontact type of speed measurement of belt conveyor has been implemented using optoelectronics. The pattern which is discussed in this paper can be easily stuck on to any rotating device such as to a roller, belt conveyor or on any other rotating surface whose rotational speed has to be measured. This pattern has a simple logic that can be easily executed by ATTINY85 controller for measuring speed. The output from the ATTINY85 is stepped up with a gain value of 2 by an operational amplifier LM358. The output voltage from the amplifier can be varied to control the speed by the available variable frequency drive. Thus, a cost effective non-contact type solution to compute the speed of the conveyor has been successfully developed with ports to easily interface with the variable frequency drives.

\section{References}

1. Hu Y, Wang L, Wang X, Qian X, Yan Y. Simultaneous measurement of conveyor belt speed and vibration using an electrostatic sensor array. IEEE International Instrumentation and Measurement Technology Conference; 2015. p. 757-610. PMCid: PMC4744654. https://doi. org/10.1109/I2MTC.2015.7151363.

2. Masaki MS, Zhang L, Xia X. A comparative study on the costeffective belt conveyors for bulk material handling. Elsevier 9th International Conference on Applied Energy; 2017. p. 2754-9. https://doi.org/10.1016/j.egypro.2017.12.221.

3. Lopera JM, Prieto MJ, Linera FF, Vecino G, Gonzalez JA. A new speed measurement system. IEEE Industry Applications Magazine. 2005; 11(6):44-51. https://doi. org/10.1109/MIA.2005.1524736.

4. He D, Pang Y, Lodewijks G. Speed control of belt conveyors during transient operation. Elsevier. Powder Technology. 2016; 301:622-31. https://doi.org/10.1016/j. powtec.2016.07.004.

5. He D, Pang Y, Lodewijks G, Liu X. Healthy speed control of belt conveyors on conveying bulk materials. Elsevier, Powder Technology. 2018; 327:408-19. https://doi. org/10.1016/j.powtec.2018.01.002. 